N.D.L.R.

Cet article de MM. Thibault et Pierre n'est pas exactement un article scientifique mais plutôt un article de vulgarisation. Toutefois, étant donné sa qualité et la valeur des enseignements qu'il contient, il nous a paru intéressant de le faire parâttre dans le B.F.P.

J.-P.C.

\title{
LE REPEUPLEMENT DU SCORFF EN SAUMON ATLANTIQUE (Salmo salar L. 1758); LE POINT SUR LES TRAVAUX ENTREPRIS
}

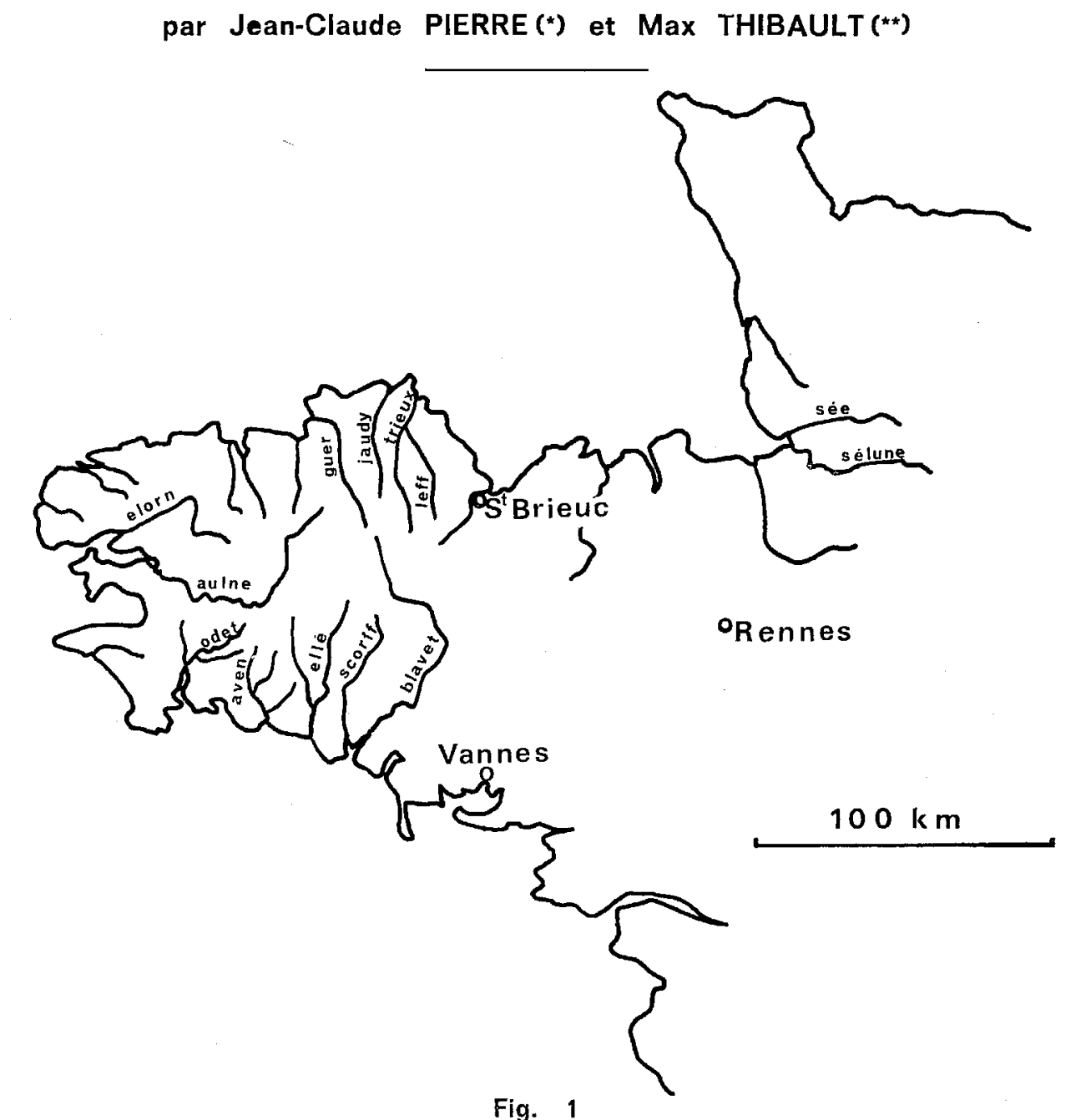

(*) Président de l'Association pour la protection du Saumon en Bretagne. Basse Normandie (A.P.P.S.B.) 1 rue des Primevères 56530 QUEVEN.

(*) Maitre Assistant, Laboratoire de Zoologie I.N.R.A. - E.N.S.A. 65 rue de St Brieuc 35042 RENNES CEDEX. 


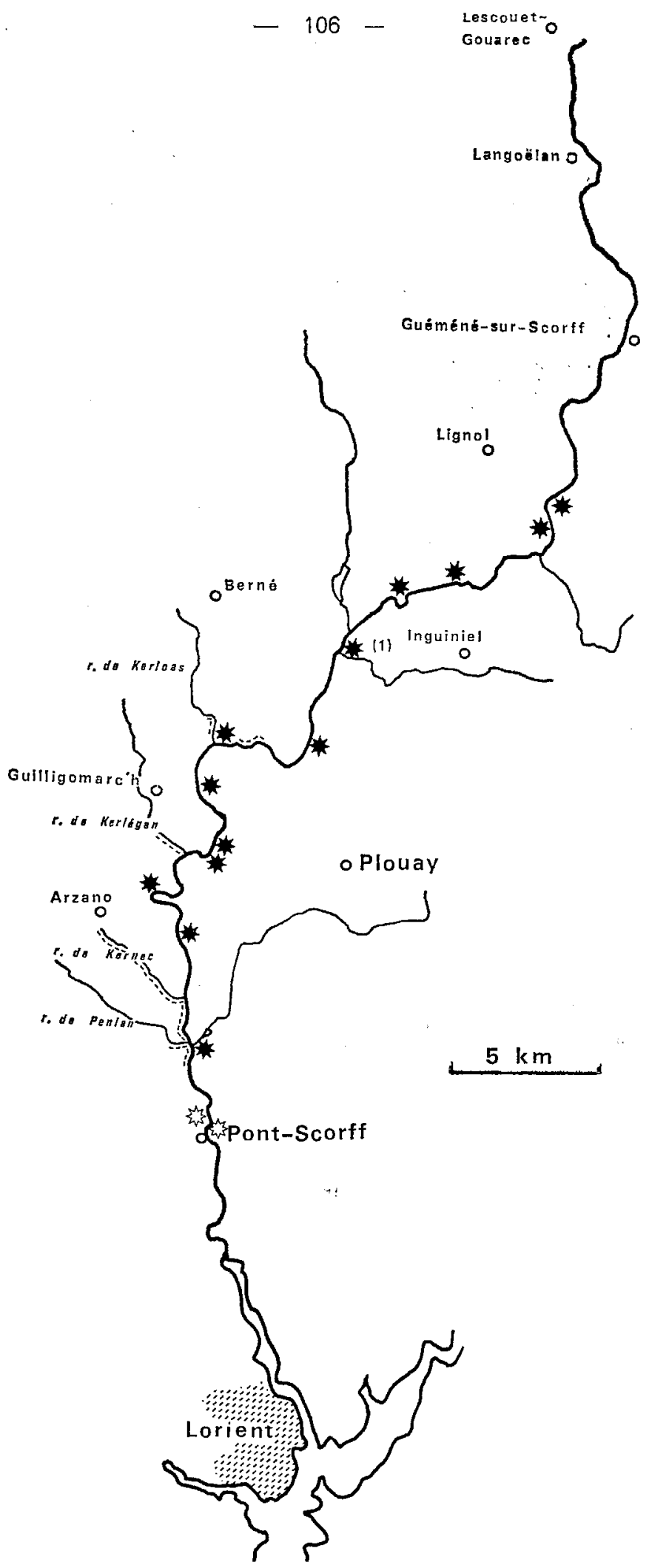

Fig. 2 


\section{INTRODUCTION}

L'action en cours sur le bassin du Scorff s'inscrit en droite ligne dans la succession d'événements qui ont marqué la vie de notre association depuis sa création en 1969 :

- première assemblée générale le 29 novembre 1970 à Carhaix;

- no 1 de la revue trimestrielle . Saumons et Truites de Bretagne et de Basse Normandie * le premier trimestre 1971;

- journée régionale d'étude sur le Saumon le 22 juin 1972 à Lorient lors de la présentation du livre blanc de I'A.P.P.S.B. : * le Saumon richesse bretonne à développer".

Une expérience devait donc être entreprise sur le terrain afin de confronter les idées aux problèmes qui se posent quotidiennement tant sur le plan pratique qu'administratif, juridique, technique, scientifique et humain. C'était la seule façon d'avancer, de trouver des solutions dans le but d'en faire profiter toutes les rivières bretonnes (Fig. 1). Pour cela, l'expérience devait être tentée sur un seul bassin, permettant ainsi la concentration des efforts afin d'avoir les meilleures chances de succès.

Une rivière a été choisie, dans le courant de l'année 1971, le Scorff, pour les raisons suivantes :

- faible longueur (64 km environ dont $12 \mathrm{~km}$ d'estuaire) (Fig. 2) proximité de l'Ellé et du Blavet (Fig. 1);

- peu de pêches privées ; deux associations de pêche et de pisciculture (A.P.P.) sur le Scorff dont seule l'A.P.P. de Plouay est pour l'instant concernée par le Saumon;

- collaboration totale entre I'A.P.P. de Plouay et I'A.P.P.S.B.;

- faibles pollutions ;

- présence d'animateurs dévoués.

Le but de cette expérience est la mise au point d'une méthodologie applicable ultérieurement à tous les cours d'eau bretons; à cet effet, l'action est menée simultanément dans les trois voies suivantes :

- amélioration de la libre circulation des poissons;

- amélioration de la qualité de l'eau;

- repeuplement.

Les problèmes posés dépassent largement le simple cours de la rivière et de ses affluents; ils concernent l'ensemble du bassin versant du Scorff. Signalons ici, la création depuis le début de l'année 1973, d'une commission : * la commission pour l'aménagement et la protection de la rivière le Scorff . Cette commission a été créée à l'initiative des Sous-Préfets de Lorient et de Pontivy; elle s'est réunie trois fois en 1973. Ont participé à ces réunions, les représentants des Administrations, les élus des communes riveraines et les Associations, tous ceux qui, de près ou de loin sont intéressés par l'eau du Scorff; parmi ces associations figurent la ligue de Bretagne de Canoë-kayak, l'A.P.P. de Plouay et l'A.P.P.S.B. pour lesquelles la présence du Saumon atlantique en quantité dans le Scorff sera le plus sûr garant de la qualité de l'eau, de cette eau qui sert aussi à l'alimentation en eau potable de l'agglomération lorientaise. 


\section{I - AMELIORATION DE LA LIBRE CIRCULATION DES POISSONS}

L'édification de barrages a été une des principales causes de la régression des populations de Saumons en France. En Bretagne ils sont en général de faible hauteur; à part ceux présents sur les deux rivières canalisées l'Auine et le Blavet (Fig. 3), ils étaient destinés à retenir l'eau pour les moulins sur la plupart des cours d'eau. Sur le Scorff il existe une vingtaine de barrages de ce type (Fig. 2) dont la majorité sont aujourd'hui désaffectés. Ils sont souvent non aménagés ou $\mathrm{ma}$ ! aménagés voire fréquemment rehaussés et non entretenus. Tout ceci rend très difficile la remontée des géniteurs vers leurs frayères. Les barrages doivent être rendus étanches et des passes doivent être construites de telle façon que les migrateurs puissent effectuer leurs déplacements en toute saison et quel que soit le niveau de l'eau.

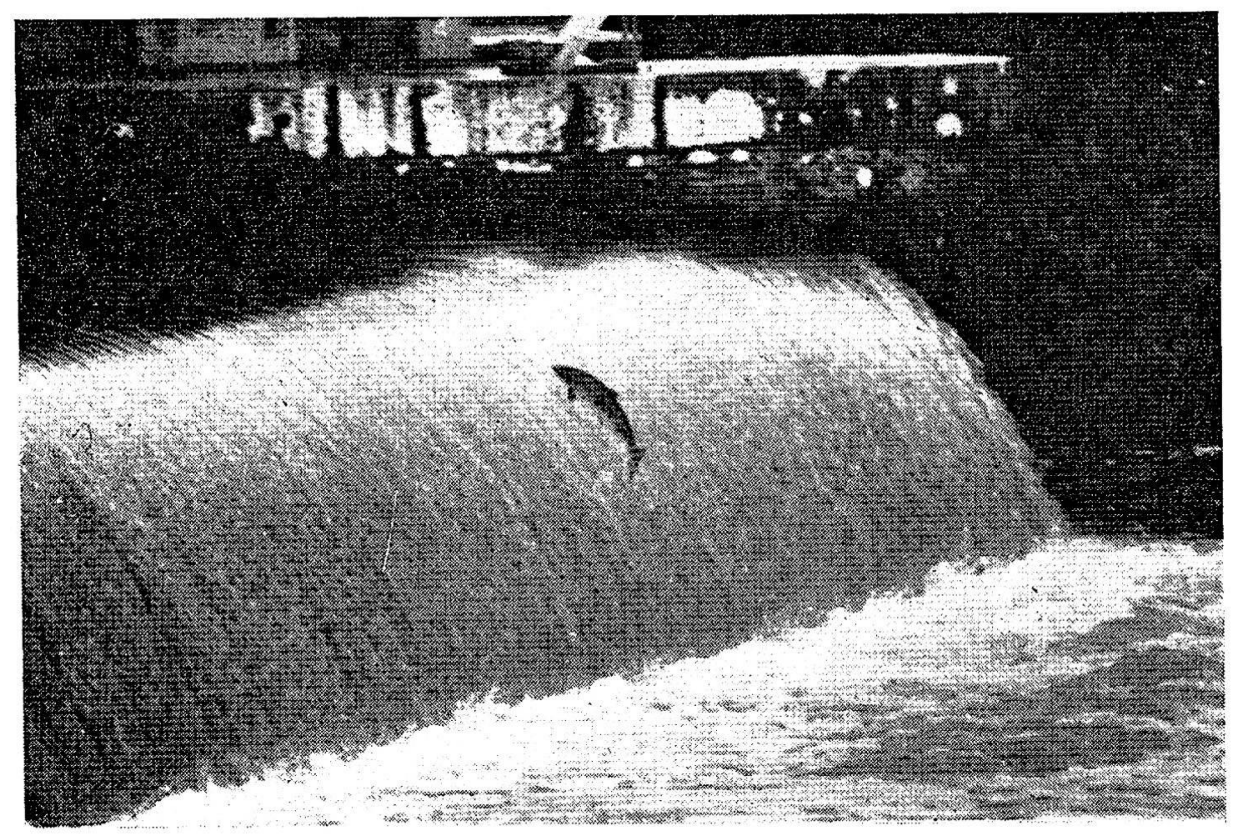

Fig. 3 - Un Saumon essaie de franchir le barrage

de Coatygrac'h sur l'Aulne à Châteaulin (juin 1972)

Comme il n'est pas possible d'aménager tous les barrages en même temps, la construction de passes se fait de l'aval vers l'amont. Rappelons ici ce qu'écrivait A. TOULLEC dans le premier numéro de la revue "Saumons et Truites " en parlant du Scorff: "l'alevinage du Scorff en Saumons ne peut se concevoir tant que l'ouvrage (Moulin des Princes, le barrage le plus en aval du Scorff) demeurera infranchissable les trois-quarts de l'année". La première passe aménagée sur le Scorff y fut construite au cours de l'automne 1971 par l'A.P.P. de Plouay, grâce à l'obligeance de Me JEGO propriétaire du barrage rive droite et grâce aux plans fournis par M. COMPAIN (1). Une seconde passe vient d'être construite dans le barrage situé immédiatement en amont du Moulin des Princes, au Moulin Leslé.

Les différents barrages du Scorff devraient être ainsi l'objet d'aménagements au cours des années à venir. 1974 verra en particulier la création d'une passe

(1) Ingénieur des Arts et Métiers, Conseiller technique de l'A.P.P.S.B. 
au barrage St-Yves. Les passes du Leslé et de St-Yves sont financées par la ville de Lorient.

\section{2 - AMELIORATION DE LA QuALITE DE L'EAU}

La qualité de l'eau du Scorff a diminué au cours de ces dernières années; cette diminution est due à la fois à certaines activités de l'homme, pollutions par exemple, et au non entretien de la rivière et des affluents.

\section{1 - Lutte contre la pollution}

Cette lutte doit se faire à deux niveaux, contre les sources actuelles de poliution (lutte curative) et contre les sources futures de pollution (lutte préventive).

L'inventaire précis des sources de pollution reste à faire sur le bassin du Scorff. II convient de préciser que l'eau du Scorff est de bonne qualité mais il faut être vigilant; les sources de pollution que nous conriaissons sont ponctuelles et pour la plupart liées à l'évolution de l'activité agricole et plus particulièrement à l'élevage (laiterie, pisciculture, etc.).

Il importe de préserver la qualité des eaux et de veiller à ce que toute implantation nouvelle soit conçue avec ce souci et que les textes réglementaires soient appliqués avec vigueur.

\section{2 - Nettoyage du Scorff et de ses affluents}

L'entretien du lit et des rives, dans la majeure partie du bassin du Scorff, n'est plus assuré depuis vingt ou trente ans. Des branches et des arbres sont tombés en travers de la rivière. (Fig. 4) obstruant son lit et entravant le libre écoulement de l'eau; ceci a eu pour effet de faciliter les inondations hivernales et de provoquer l'envasement progressif de la riviere.

Ce processus a été accéléré au cours de ces dernières années; les arbres et les souches des talus ont parfois été poussés vers la rivière lors des opé-

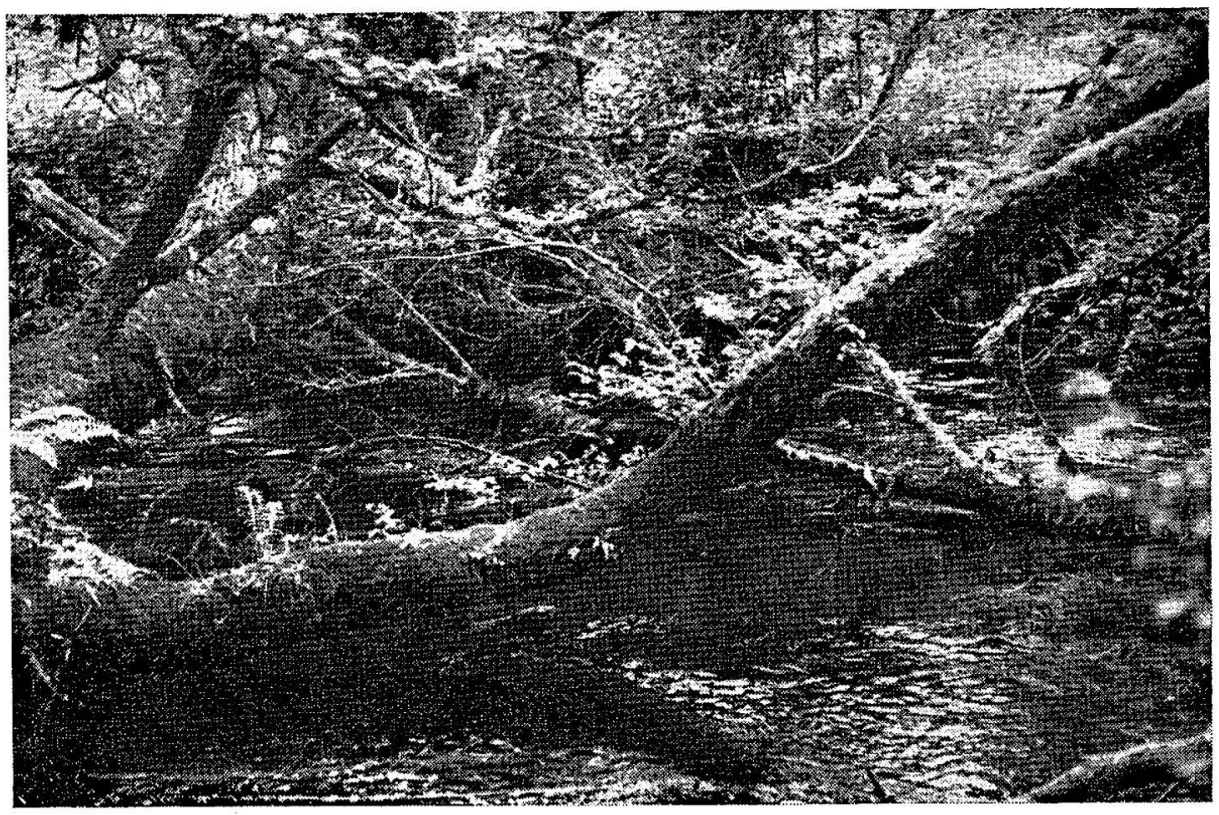

Fig. 4 - Etat de certaines portions du Scorff

(entre Pont-Scorff et le barrage de Pont-Calleck) avant le nettoyage 
rations connexes au remembrement, accentuant encore l'envasement. Enfin, il faut y ajouter le déversement de déchets de toutes sortes par l'homme qui considère encore trop souvent un cours d'eau comme une poubelle ambulante.

Cet envasement a recouvert les cailloux du fond, diminuant ainsi la surface des frayères, la quantité d'abris disponibles pour les jeunes Salmonides et la source de production de larves d'insectes aquatiques dont se nourrissent la Truite et le Saumon au cours de leurs premières années de vie.

Par ailleurs, les arbres des rives, forment une voûte presque impénétrable aux rayons du soleil, surtout dans les zones à courant ralenti ; ils inhibent ainsi le développement des plantes aquatiques d'eau courante qui, non seulement oxygènent l'eau, mais aussi servent de support aux insectes aquatiques et d'abri aux poissons.

La rivière non entretenue, se stérilise lentement mais progressivement. En d'autres termes le non entretien peut être assimilé à une pollution (2). De gros poissons peuvent encore être présents mais la reproduction va en diminuant. Dans ces conditions, les réalevinages avec des truitelles de pisciculture, déjà fort aléatoires par ailleurs, ne peuvent être qu'inopérants et illusoires compte tenu de l'état de l'habitat. Pour reprendre l'expression d'un pêcheur local, « il est aussi ridicule d'aleviner un tel cours d'eau que d'ensemencer un champ en friche".

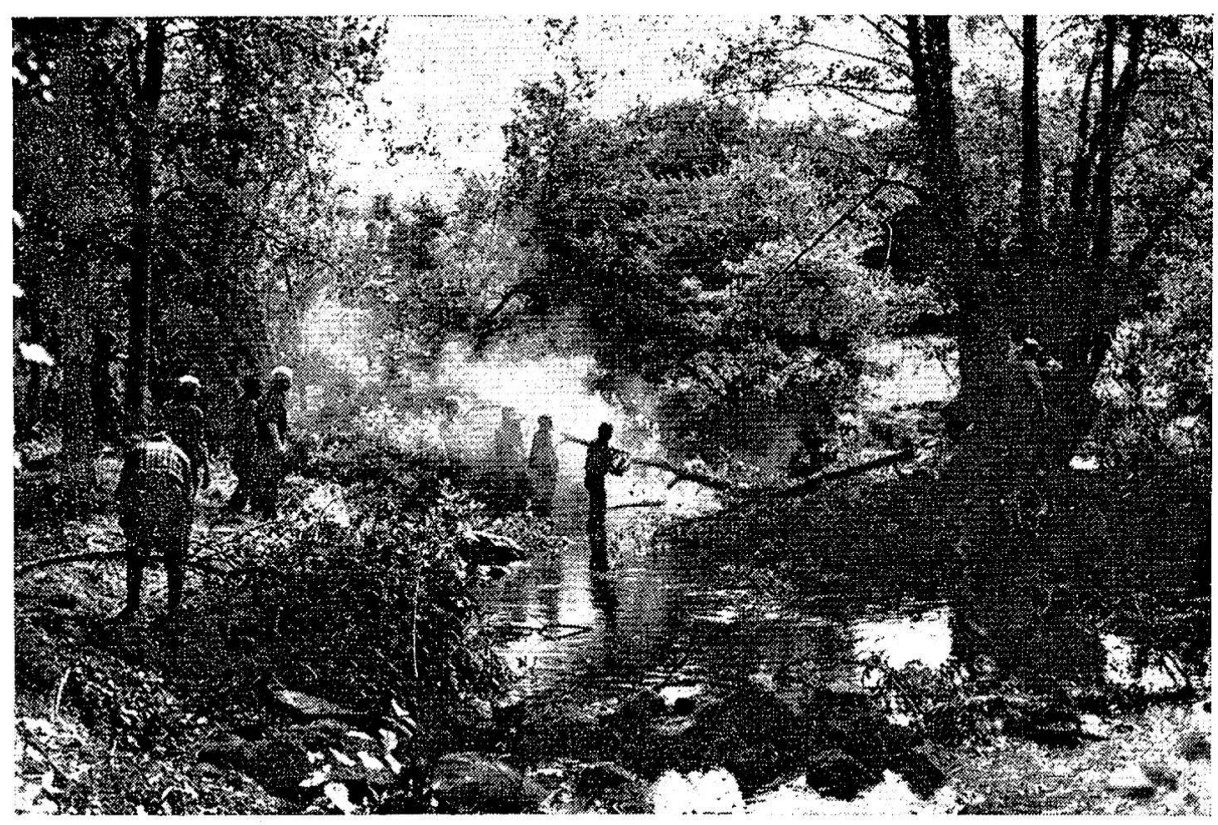

Fig. 5 - Travaux de nettoyage dans le Scorff en amont du confluent avec le ruisseau de Kerloaz au site de Pontulaire

Juridiquement, l'entretien des rives et du lit de la rivière est à la charge des riverains. La plupart de ceux-ci sont agriculteurs; or, compte tenu de l'évo-

(2) La pollut!on des eaux se rapporte à des "déversements, écoulements, rejets, dépôts directs ou indirects de matières de toute nature et plus généralement à tout fait susceptible de provoquer ou d'accroitre la dégradation cies eaux en modifiant leurs caractéristiques physiques, chimiques, biologiques, ou bactériologiques, qu'il s'agisse d'eaux superficielles, souterraines ou des eaux de la mer dans la limite des eaux territoriales n (Definition, Loi $n^{\circ}$ 64-1245 du 16 décembre 1964, Titre I, Art. 1 ${ }^{\mathrm{er}}, \S$ 6) (in ARRIGNON, 1970). 
lution de l'agriculture (mécanisation et diminution du nombre d'agriculteurs) depuis une vingtaine d'années, ces agriculteurs ne sont plus assez nombreux et n'ont plus le temps d'effectuer ce travail.

Cet entretien devrait être à la charge de tous les utilisateurs d'eau, qu'il s'agisse de ceux qui utilisent l'eau comme eau potable (habitants des communes riveraines), comme moyen de travail (pisciculture, industries lagro-alimentaires, etc.) ou comme loisirs (promeneurs, pêcheurs et adeptes du Canoë-kayak), tous en effet sont concernés par l'entretien des rivières et leur bon état.

Les opérations de nettoyage (Fig. 5) ont débuté à l'initiative de l'A.P.P.S.B. et I'A.P.P. de Plouay le 31 juillet 1972. Le nettoyage s'est poursuivi régulièrement au cours des différents week-ends à la fois sur la rivière principlale et sur quatre de ses affluents rive droite. $Y$ ont participé, non seulement les pêcheurs mais également les bénévoles amis de la nature et les chercheurs et étudiants de I'Université et de l'Ecole Nationale Supérieure Agronomique (E.N.S.A. de Rennes). En 1973, ce travail s'est poursuivi et amplifié au cours des vacances d'été grâce au concours de nombreux jeunes intéressés à l'opération Scorff et dépendant de l'Association "Etudes et Chantiers". La Marine Nationale a également commencé d'apporter son aide laux travaux en cours en faisant participer des unités de "commandos" aux opérations de nettoyage.

Il faut signaler qu'un trop petit nombre de pêcheurs (de plus, toujours les mêmes) ont participé à ces travaux de nettoyage: peut-être une centaine au maximum bien que l'A.P.P. de Plouay compte environ 1500 membres dont 200 pêcheurs de Saumon. Or, les opérations de remise en valeur du Scorff concernent aussi bien la Truite que le Saumon. Dans l'éditorial "Objectif 2000 " du no 1 de notre bulletin, nous écrivions: "ces deux cousins germains (Truite et Saumon) ont des exigences identiques en ce qui concerne la pureté de l'eau, la qualité des frayères, et tout ce qui sera fait pour l'un sera bénéfique pour l'autre". Ceci est confirmé par les résultats des premières études (Cf. chapitre 4).

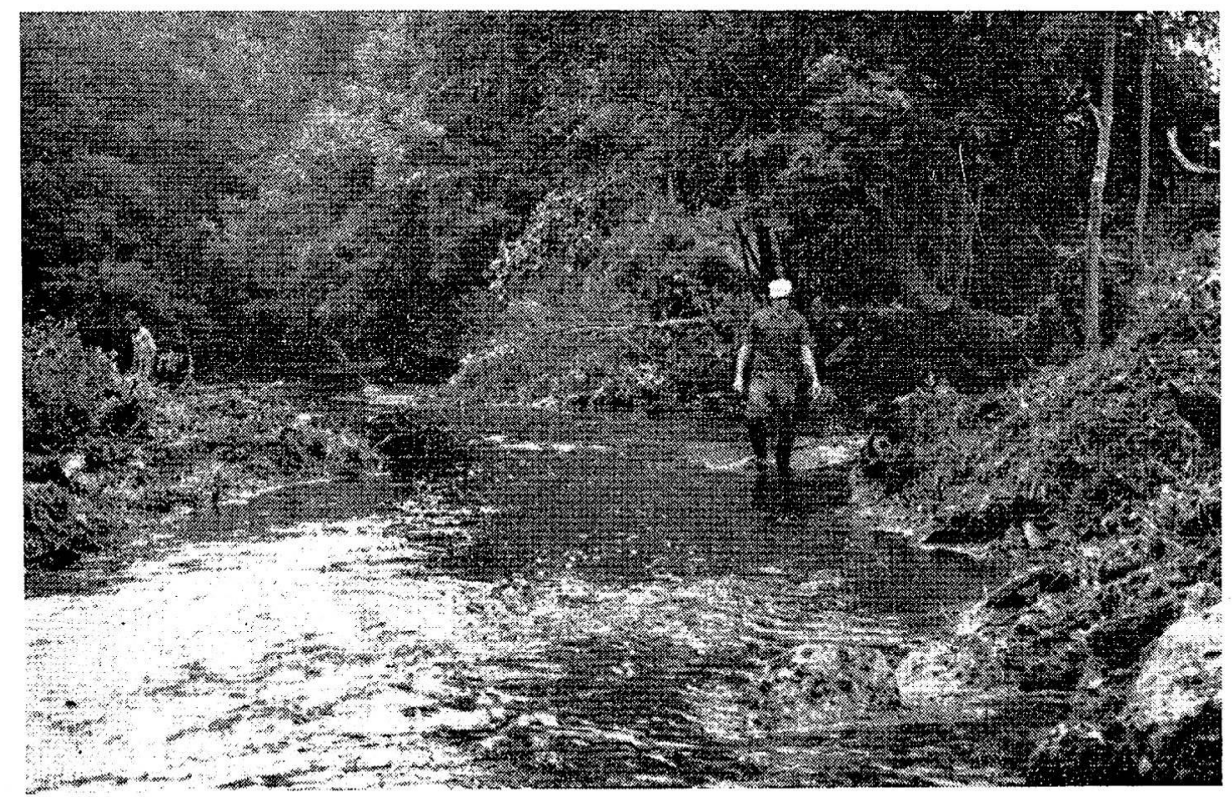

Fig. 6 - Travaux de nettoyage dans le Scorff entre le confluent avec le ruisseau de Kernec et le confluent avec le ruisseau de Penlan. 
Tous les arbres et branches immergés sont retirés de la rivière, hissés sur la rive à l'aide de cordes, débités à la hache et à la tronçonneuse et brûlés immédiatement. Les arbres des rives sont élagués, éclaircis, les ronces sont coupées (Fig. 6). Un peu plus d'un an après le début des travaux, environ $4 \mathrm{~km}$ d'une rive du Scorff et $6 \mathrm{~km}$ des deux rives d'affluents ont été nettoyés (Fig. 2).

Pour les ruisseaux, des sections de 500 à $700 \mathrm{~m}$ dans les parties aval de chacun d'eux ont été nettoyées en $1972(2,5 \mathrm{~km})$; en 1973 , y ont été ajoutés $500 \mathrm{~m}$ sur un ruisseau (Kerlégan) et $3,5 \mathrm{~km}$ sur le ruisseau de Kernec jusqu'à proximité de sa source, au hameau du Moustoir près d'Arzano. La durée du travail pour le nettoyage de ces ruisseaux a été de l'ordre de 1000 heures en 1972 et de 1400 heures en 1973 ; ce qui représente une moyenne de 25 heures pour 100 mètres de ruisseau. L'automne 1973 a aussi été consacré à l'entretien des $2,5 \mathrm{~km}$ nettoyés l'année précédente; cet entretien a demandé dix fois moins de temps et peut être évalué à 2 heures et demie pour 100 mètres de ruisseau.

Après ces opérations de nettoyage, des travaux d'aménagement ont été réalisés sur trois ruisseaux au cours de l'hiver 1972-73 et sont en cours sur le ruisseau de Kernec: des cailloux sont transportés et amenés sur les rives. lls sont ensuite immergés, soit en tas pour constituer de petits barrages tous les dix mètres environ, soit dispersés sur le fond afin de diversifier l'habitat. La construction de barrages permet de relever le niveau d'eau en amont du barrage tout en provoquant une zone de courant fortement oxygénée en aval. L'apport de cailloux entre chaque barrage est destiné à la fois à augmenter la quantité d'abris et d'insectes vivant sur les cailloux pour les poissons.

Nous tenons à remercier tous les riverains qui nous ont facilité la tâche et qui souvent nous ont donné un a coup de main" en prêtant la tronçonneuse ou bien le tracteur, voire en participant aux travaux. Ces derniers ne sont pas réalisés seulement pour les pêcheurs mais aussi pour les agriculteurs; la bonne qualité de l'eau, indispensable pour le développement des Salmonides l'est également pour le bétail.

\section{3 - REPEUPLEMENT}

Le repeuplement a débuté au printemps 1973. Pour ce faire, des œufs de Saumon atlantique ont été importés d'Ecosse par le C.N.E.X.O.; la fin de l'incubation et le début de la résorption de vésicule ont été assurés à la pisciculture de M. CALMELS dans les Côtes-du-Nord.

Un premier essai de transport et d'immersion concernant environ 6000 alevins a été tenté le 5 avril 1973. Le reste des alevins $(60000)$ a été immergé deux jours plus tard.

Les alevins sont mis dans des sacs en matière plastique contenant environ 5 litres d'eau. L'oxygène est insufflé à la partie supérieure du sac qui est ensuite solidement fermé. Compte tenu de la température de l'air à cet époque, les sacs ont été mis côte à côte dans le coffre des voitures sans précaution spéciale pour le maintien d'une basse température. Le transport et l'immersion ont été assurés par les membres de l'A.P.P.S.B., les chercheurs et les étudiants de l'E.N.S.A. de Rennes.

Les sacs, répartis le long du ruisseau sont laissés en contact de l'eau pendant une demi-heure à deux heures afin que la température de l'eau du sac s'équilibre avec la température de l'eau du ruisseau. Ensuite le sac est versé délicatement dans un entonnoir en polyéthylène dont la partie infé- 
rieure conduit les alevins à l'intérieur d'une frayère artificielle. Cette dernière est constituée d'un petit tas de cailloux immergés environ une semaine avant la mise des alevins.

\section{4 - ETUDES ECOLOGIQUUES}

Parallèlement à ces travaux de nettoyage, d'aménagement et de repeuplement entrepris sur le Scorff, des travaux de recherche sont menés dans le cadre d'un progamme d'étude: "Ecologie et Aménagement des rivières à Saumon de Bretagne "; ce programme financé par la Délégation Générale à la Recherche Scientifique et Technique (D.G.R.S.T.) et par I'Institut National de la Recherche Agronomique (I.N.R.A.), comporte deux volets, d'une part étude des populations de Saumon, d'autre part étude écologique de quatre affluents du Scorff. Ces différentes recherches sont effectuées par des chercheurs et des étudiants de I'Université et de I'I.N.R.A.-E.N.S.A. de Rennes.

\section{1 - Etude des populations de Saumon}

Elle peut être qualifiée d'étude à court terme; ébauchée en 1971, elle s'est surtout développée à partir de 1972 et doit se terminer à la fin de 1974 . Elle est destinée à obtenir le maximum de renseignements concernant l'écoéthologie du Saumon atlantique en Bretagne puisqu'on ne disposait d'aucune donnée scientifique sur ce poisson en Bretagne au début de l'étude. Cette dernière comprend deux aspects :

- la descente des smolts,

- la remontée des adultes.

\section{2 - Etude écologique de quatre affluents du Scorff}

Il s'agit ici d'une étude à long terme qui a débuté en 1972. Elle doit déboucher sur la mise au point d'une technique de repeuplement en Saumon atlantique à l'aide de ruisseaux pépinières, applicable ultérieurement à tous les cours d'eau bretons.

Les premiers échantillonnages, inventaires et analyses ont été effectués avant les opérations de nettoyage et d'aménagement. Ont été mises en évidence les principales caractéristiques :

- de la physico-chimie de l'eau des ruisseaux,

- des différentes espèces végétales aquatiques,

- du substrat sédimentologique,

- du peuplement animal, en invertébrés et en poissons.

A partir de 1973, quatre thermographes ont été mis en place sur les quatre ruisseaux. Les échantillonnages sont poursuivis afin de suivre l'influence des travaux entrepris (élaguage des arbres des rives, nettoyage du lit du ruisseau, apport de cailloux) sur la végétation, le substrat (en particulier déplacement des sédiments fins) et le peuplement animal en invertébrés (surtout les insectes aquatiques à stades adultes aériens) et en poissons.

Les inventaires des populations de poissons sont réalisés à l'aide de deux à quatre pêches électriques successives. Les Anguilles et les Truites ont été enlevées dans les portions aménagées (500 à $700 \mathrm{~m}$ de longueur de chaque ruisseau), d'une part lors des inventaires réalisés à l'automne 1972, d'autre part lors d'une pêche électrique réalisée à la fin d inventaires effectués à l'automne 1973 ont eu pour but de suivre l'évolution dú 
peuplement piscicole et l'implantation en Saumon atlantique suite à cet enlèvement des populations et à l'immersion des jeunes saumons effectuée au début du mois d'avril.

Anguille : Anguilla anguilla (L., 1758)

La densité de population et la biomasse d'anguilles sont à un niveau beaucoup plus bas qu'en 1972, respectivement 0,3 à 0,4 individu $/ \mathrm{m}^{2}$ en 1972 et 0,07 à 0,1 individu $/ \mathrm{m}^{2}$ en 1973 et 16 à $21 \mathrm{~g} / \mathrm{m}^{2}$ en 1972 et 0,25 à $4,35 \mathrm{~g} / \mathrm{m}^{2}$ en 1973 .

Truite commune : Salmo trutta (L., 1758)

Dans presque tous les cas (sauf un sous-secteur d'ụn ruisseau à faible pente), la reconstitution de la population de Truites aboutit à une densité de population supérieure à celle observée à l'automne 1972 et ceci, précisons-le, sans alevinage : 0,08 à 0,38 individu $/ \mathrm{m}^{2}$ en 1972 et 0,13 à 0,60 individu $/ \mathrm{m}^{2}$ en 1973 . Les variations de la biomasse sont faibles, le plus souvent en légère augmentation : 4 à $5,82 \mathrm{~g} / \mathrm{m}^{2}$ en 1972 et 3,81 à $7,90 \mathrm{~g} / \mathrm{m}^{2}$ en 1973

Saumon atlantique : Salmo salar (L., 1758)

La densité de population des jeunes Saumons est plus élevée si le ruisseau a été nettoyé et si les autres poissons ont été enlevés. Elle est également plus élevée si le ruisseau a une pente supérieure à $1 \%\left(0,13\right.$ et 0,21 individu $/ \mathrm{m}^{2}$ pour deux ruisseaux); dans les deux autres ruisseaux où la pente est inférieure à $1 \%$, la densité atteint seulement 0,05 et 0,09 individu $/ \mathrm{m}^{2}$. Les jeunes Saumons se sont déplacés vers l'amont et surtout vers l'aval de leur point d'immersion.

La croissance est la meilleure lorsque la densité est la plus faible; mesurant $25 \mathrm{~mm}$ au début de leur alimentation, ces poissons atteignaient, six mois plus tard, 69 a) $117 \mathrm{~mm}$ dans un ruisseau (densité : $0,05 / \mathrm{m}^{2}$ ) et 59 à $101 \mathrm{~mm}$ dans un autre ruisseau (densité : $0,21 / \mathrm{m}^{2}$ ).

\section{CONCLUSION}

Chacun le sent confusément, la pèche des Salmonides en France se trouve à un tournant. II est vain d'espérer que la nature réparera d'elle-même les outrages que lui inflige l'homme mais vain aussi d'espérer que l'on pourra retrouver nos richesses salmonicoles passées en laissant le milieu actuel continuer de se dégrader chaque jour davantage.

Trop de pêcheurs et trop de dirigeants des associations de pêche s'imaginent encore qu'il est possible de capturer toujours plus de poissons sur un parcours pourvu qu'on y mette le prix; c'est-à-dire qu'on y déverse chaque année un peu plus de truitelles ou de truites de bonne taille. Ces réempoissonnements à partir de sujets issus de pisciculture, mal adaptés aux dures lois de la vie sauvage, réalisés le plus souvent à une mauvaise période, sont un leurre; bien pis, ils risquent de causer un préjudice au patrimoine naturel en y introduisant diverses maladies (parasitaires, microbiennes, virales).

La gestion d'un patrimoine piscicole ne peut se faire :

- sans la restauration de l'habitat puis son entretien;

- sans l'acquisition de connaissances écologiques;

- sans l'application des textes législatifs actuels et la modification de la législation dans certains cas.

A tous ces égards, l'expérience en cours sur le Scorff nous apporte des enseignements précieux :

- les travaux de nettoyage et la limitation des populations de prédateurs dans les ruisseaux ont eu un effet rapide et immédiat sur les jeunes Salmonides ; 
ils ont permis dans certains cas le doublement des populations en une seule année.

- la croissance des Salmonides dans les rivières bretonnes est bonne (BAGLINIERE et FONTENELLE, 1972; LUSSIAA-BERDOU, 1972, EUZENAT et LEGLIZE 1973), comparable à la moyenne nationale (CUINAT, 1971); les poissons atteignent $23 \mathrm{~cm}$ à trois ans malgré la faible teneur en calcium des eaux ( 2 à 15 $\mathrm{mg} / \mathrm{l}$; BERTRU, communication personnelle). Autoriser les captures à la taille de $18 \mathrm{~cm}$ (limite actuelle légale de la taille de capture) c'est tuer le blé en herbe; la plupart de ces truites sont âgées d'un an et n'ont pu encore se reproduire.

- la police des eaux est totalement à revoir. Actuellement les remontées des migrateurs, de même que le bon déroulement de la fraie sont soumis au seul bon vouloir de certains utilisateurs d'eau ou riverains; ces derniers manœuvrent leurs vannes et modifient les niveaux d'eau sans le moindre souci des règles écologiques ou de l'application de la loi.

De nouvelles règles de gestion de nos ressources piscicoles, mais également de nos ressources en eau douce d'une façon générale, sont à mettre en œuvre. II n'est plus possible de découper la rivière et ses affluents en tranches, selon l'A.P.P. (3) ou le département. Une gestion efficace doit se faire ä l'échelle du bassin versant et passe dans un premier temps par la connaissance de ce bassin. C'est pourquoi, compte tenu du caractère expérimental du Scorff grâce aux études hydrobiologiques qui ont débuté en 1972, deux nouvelles études viennent de démarrer :

- étude hydrologique du bassin versant du Scorff (4) depuis la fin de l'année 1973. Un programme de trois ans a été mis sur pied; son financement est assuré par une aide de la rénovation rurale (Délégation à l'Aménagement du Territoire et à l'Action Régionale: D.A.T.A.R.). L'étude sera réalisée à la fois au niveau du réseau hydrographique et au niveau du bassin versant.

(3) II est regrettable de constater que les différentes A.P.P. qui jalonnent une même rivière peuvent avoir des politiques radicalement différentes, voire opposées; c'est ainsi que certaines A.P.P. alevinent en Salmonides alors que leurs voisines immédiates le font en prédateurs de Salmonides.

\section{(4) ETUDE HYDROLOGIQUE.}

1) Au niveau du réseau hydrographique. Cette première partie de l'étude a pour objet la détermination de l'importance et du rôle des diverses retenues d'eau du Scorff puis des aménagements à réaliser pour faciliter la libre circulation du Saumon atlantique.

2) Au niveau du bassin versant. Cette seconde partie comporte deux aspects :

aspect descriptif : les facteurs humains et de l'utilisation des sols (importance de la couverture végétale; forêts, bocage, type de culture) devront être déterminés ainsi que le complexe physique du bassin.

aspect dynamique : l'étude du fonctionnement de l'écoulement de l'eau sera entreprise au niveau de petits bassins expérimentaux. Les fluctuations de débit d'eau sseront étudiées en liaison avec la pluviométrie au niveau de l'averse pour connaitre la dynamique des eaux : ruissellement et infiltration. Cette étude comprendra également la surveillance et l'évolution des nappes phréatiques ainsi que l'analyse d'échantillons d'eau prélevés à intervalles réguliers. 


\section{- étude socio-économique du bassin versant du Scorff (5).}

Elle débute en 1974 grâce à l'aide financière de la D.G.R.S.T. Un travail général couvrant les principaux aspects socio-économiques du bassin versant $\mathrm{du}$ Scorff est envisagé. II importe de rattacher les problèmes de nature purement hydrobiologique de la qualité des eaux du Scorff à l'ensemble des activités de la zone, plus particulièrement en ce qui concerne l'agriculture et les Salmonides.

L'A.P.P.S.B. I'a démontré dans son livre blanc (Anonyme 1972), la pêche du Saumon et de la Truite peut constituer un atout exceptionnel dans le développement d'un tourisme d'avant saison en Bretagne. Máis la bonne volonté ne suffit plus et l'expérience du Scorff le prouve, la gestion de la richesse régionale que constituent les Salmonides implique la présence de scientifiques de haut niveau; compte tenu du contexte écologique particulier des rivières du massif armoricain deux voies complémentaires doivent être envisagées : région ;

- création d'un centre de recherches salmonicoles au niveau de la

- application de ces recherches sur chaque bassin versant grâce à la présence d'un écologiste formé aux techniques salmonicoles et à l'entretien des rivières.

Ecologiste piscicole, une nouvelle carrière? Pourquoi pas.

\section{REMERCIEMENTS}

Tout ce travail n'a pu être réalisé sur le Scorff que grâce à la bonne volonté et à l'esprit de collaboration de tous ceux qui, de près ou de loin, se sentent concernés par l'expérience entreprise :

- les pêcheurs, les membres de l'A.P.P.S.B., les riverains, les bénévoles, scientifiques ou non qui, depuis le 31 juillet 1972, se retrouvent chaque week-end ou pendant leurs congés sur le Scorff;

- I'A.P.P. de Plouay, la Fédération des A.P.P. du Morbihan et la Région piscicole de l'Ouest dont les gardes-pêche participent aux inventaires piscicoles effectués à l'aide de la pêche électrique.

\section{(5) ETUDE SOCIO-ECONOMIQUE.}

Le programme des travaux comporte deux volets :

1) Evolution de l'activité agricole : la mise en évidence de l'évolution de l'agriculture depuis une quinzaine d'années permettra de savoir, d'une part si cette zone est marquée ou non par un exode agricole et rural plus important qu'ailleurs, d'autre part si cette diminution de population a une influence sur les systèmes de production.

2) Problemes hydrobiologiques. Ils sont de deux ordres :

liés à la pollution : recensement des sources de pollution et chiffrer leur importance selon le type d'effluents;

liés à l'aménagement en vue du repeuplement en Saumon atlantique : afin de déterminer l'importance socio-économique de la pêche au Saumon et à la Truite sur le Scorff, il s'avère nécessaire d'étudier la population des pêcheurs en distinguant les pêcheurs extérieurs à la région et les pêcheurs de la région.

Un des intérêts de cette étude réside dans le fait qu'elle permettra de connaitre les divers aspects socio-économiques de la pêche au Saumon et à la Truite avant que les travaux actuellement en cours (nettoyage, aménagement et repeuplement) aient comméncé à porter leurs fruits. II sera intéressant de suivre l'évolution des diverses catégories de pêcheurs dès que des modifications d'ordre hydrobiologique seront apparues. 


\section{- BIBLIOGRAPHIE SOMMAIRE -}

Anonyme 1968 - L'eau aperçu général - Région Bretagne comité technique de l'eau. $15 \mathrm{p}$.

Anonyme 1972 - Le Saumon richesse bretonne à développer. Livre blanc A.P.P. S.B., $54 \mathrm{p}$.

ARRIGNON J. 1970 - Aménagement piscicole des eaux intérieures. S.E.D.E.T.E.C. S.A. Edit. Paris, 1 vol., 643 p.

BAGLINIERE J.-L., FONTENELLE G., 1972 - Recherches préliminaires sur le Saumon atlantique (Salmo salar L., 1758) en Bretagne D.A.A.-P.A.M.N. et D.E.A. Eco-éthologie E.N.S.A. et Université de Rennes, 42 p.

CUINAT R., 1971 - Principaux caractères démographiques observés sur 50 rivières à truites françaises. Influence de la pente et du calcium. Ann. Hydrobiol., 2, 2, 187-207.

EUZENAT G., LEGLIZE L., 1973 - Etude hydrobiologique du Blavet (Morbihan). D.A.A.-P.A.M.N. et D.E.A. Eco-éthologie E.N.S.A. et Université de Rennes, $81 \mathrm{p}$.

LUSSIAA-BERDOU J.-P., 1972 - Ecologie d'un ruisseau à Salmonides du Morbihan : les populations de poissons. D.A.A. Halieutique E.N.S.A. de Rennes, $32 \mathrm{p}$. 


\section{$-118-$ \\ - LEGENDE DES FIGURES -}

Fig. 1 - Rivières à Saumon atlantique de Bretagne et de Basse-Normandie.

On remarque la forte densité de ces rivières en Bretagne occidentale (à l'Ouest de la ligne Vannes-St-Brieuc) par rapport à la Bretagne orientale située à l'est de cette ligne. Cette différence peut être expliquée par les caractéristiques suivantes (Anonyme, 1968) : Bretagne occidentale - forte pluviosité $(800 \mathrm{~mm}$ sur le littoral à environ $1500 \mathrm{~mm}$ au cœur des monts d'Arrée) due à sa profonde pénétration en mer, sous-sol granitique, relief accusé et étiages soutenus par une multitude de petites sources.

Bretagne orientale - pluviométrie plus faible $(900 \mathrm{~mm}$ juste après la ligne Vannes-St-Brieuc à $700 \mathrm{~mm}$ au centre du bassin de Rennes), sous-sol surtout schisteux, relief moins accusé et étiages plus prononcés en été ; les rivières, lentes, sont surtout classées en seconde catégorie.

En Basse-Normandie - les rivières à Salmonides sont de nouveau présentes car on retrouve les principales caractéristiques de la Bretagne occidentale, quoique moins accentuées.

Fig. 2 - Le Scorff et ses principaux affluents; seuls les quatre affluents de la rive droite, en cours de transformation en ruisseaux pépinières, sont nommés.

Le Scorff est une rivière d'eau douce seulement à partir de Pont-Scorff; en effet, les marées se font sentir jusqu'à cette ville. Sont indiqués sur cette carté, les barrages sur la rivière principale (astérisques) et les portions nettoyées (tirés) tant sur le Scorff luimême que sur les affluents.

Barrages - en astériques clairs, les barrages maintenant rendus étanches et possèdant des passes à poissons migrateurs; en astériques sombres, les barrages qui restent à aménager. Pour l'instant, le barrage marqué (1) peut être considéré comme difficilement franchissable par le Saumon; autrement dit, la fraie est possible sur une portion de rivière d'environ $23 \mathrm{~km}$.

Portions nettoyées - au cours d'une période de 17 mois (31 juillet 1972 au 31 décembre 1973); en général une, seule rive a été nettoyée sur le Scorff $(4 \mathrm{~km})$ alors que les deux rives le sont sur les affluents $(6 \mathrm{~km})$.

\section{AMELIORATION DE LA LIBRE CIRCULATION DES POISSONS}

Fig. 3 - Les barrages des rivières canalisées.

Les deux plus longues rivières à Saumon de. Bretagne (Aulne et Blavet) sont canalisées sur une partie de leurs cours. Ces barrages sont difficiles à franchir à la fois parce que des réhausses ont été fréquemment installées et parce que la fosse d'appel en bas de la lame déversante est insuffisamment profonde; c'est ainsi que sur près de 1600 sauts observés à l'écluse de Quellenec sur le Blavet des mois d'avril à août 1973, seulement 3 à $10 \%$ des sauts sont réussis. L'efficacité réêlle des passes, quand elles existent et quand elles sont correctement alimentées en eau, reste à démontrer.

\section{AMELIORATION DE LA QUALITE DE L'EAU}

Fig. 4 - état de certaines portions du Scorff (entre Pont-Scorff et le barrage de PontCalleck) avant le nettoyage.

Fig. 5 - travaux de nettoyage dans le Scorff en amont du confluent avec le ruisseau de Kerloaz au site de Pontulaire.

Fig. 6 - travaux de nettoyage dans le Scorff entre le confluent avec le ruisseau de Kernec et le confluent avec le ruisseau de Penlan. 